\title{
Diffractive X-ray Optics for Synchrotrons and Free-Electron Lasers
}

$\underline{\text { Christian David }}^{1}$, Benedikt Rösner ${ }^{1}$, Florian Döring ${ }^{1}$, Vitaliy Guzenko ${ }^{1}$, Frieder Koch $^{1}$, Maxime Lebugle $^{1}$, Felix Marschall ${ }^{1}$, Gediminas Seniutinas ${ }^{1}$, Jörg Raabe ${ }^{1}$, Benjamin Watts ${ }^{1}$, Daniel Grolimund ${ }^{1}$, Zhong Yin ${ }^{2,3}$, Martin Beye ${ }^{3}$, Simone Techert ${ }^{2,3,4}$, Jens Viefhaus ${ }^{3}$, Gerald Falkenberg ${ }^{3}$, Christian Schroer ${ }^{3}$

1. Paul Scherrer Institut, CH-5232 Villigen, Switzerland

2. Georg-August Universität Göttingen, 37077 Göttingen, Germany

3. Deutsches Elektronen Synchrotron, D-22607 Hamburg, Germany

4. Max Planck Institute for Biophysical Chemistry, 37077 Göttingen, Germany

* Corresponding author, christian.david@psi.ch

$\mathrm{X}$-rays are excellent probes for the investigation of matter using scattering, imaging and spectroscopic techniques, offering high penetration capability, spatial and temporal resolution, along with elemental and chemical sensitivity. Accelerator-based photon sources such as synchrotrons and X-ray free-electron lasers (XFELs) play a key role in these analytical techniques as they offer beams with unique brilliance. At the Paul Scherrer Institut, Switzerland, we work on the developments of X-ray instrumentation and experimental techniques based on diffractive optics. These optical elements are designed for short wavelength radiation ranging from the vacuum ultraviolet to hard X-rays. In contrast to other kinds of $\mathrm{X}$-ray optics such as mirrors and lenses, diffractive optics allow for precise control of the optical wave front and the realization of complex optical functionalities. Recent examples for this are X-ray beam shapers as illumination systems in full-field X-ray microscopes [1] or spiral zone plates that can produce XFEL pulses carrying an angular momentum [2].

The key challenges lie in the fabrication of the diffractive structures by advanced nanolithography techniques, as they need to provide dimensions and placement accuracies down to the nanometer scale. We apply high energy electron-beam lithography to produce resist patterns with high aspect ratios that can be transferred into materials that provide a suitable X-ray refractive index in order to obtain good diffraction efficiencies. In X-ray microscopes, Fresnel zone plates (FZPs) are used as lenses to produce high resolution images. As the resolution of a FZP is essentially limited to the width of its smallest, outermost zones, extensive work has been invested to reduce the zone width. Conventional lithography approaches break down in the range of around $20 \mathrm{~nm}$, and advanced nanofabrication schemes need to be applied to reach the $10 \mathrm{~nm}$ resolution regime. A line-doubling approach has been developed that is based on the electron-beam writing of sparse template structures and subsequent deposition of the zone plate material by atomic layer deposition (ALD) [3-5]. This method leads to a doubling of the zones, and consequently, to a significant increase in resolution.

In order to apply this scheme to soft X-ray scanning microscopy, it is essential to further develop this method as shown in Figure 1. In addition to the first two fabrication steps, the resist patterning and the coating by ALD, we have used Argon ion milling to remove the metal layer on top of the zone structures in order to be able to remove the resist material between the metal structures by hydrofluoric acid vapor. This is essential to avoid absorption losses which would strongly reduce the diffraction efficiency of line-doubled FZPs when used in the soft X-ray range. Figure 1 shows the structures of a line-doubled FZP with $8.8 \mathrm{~nm}$ zone width at various stages during its fabrication process [6]. Such FZPs have been tested at the Scanning Transmission X-ray Microscope (STXM) at the PolLux beam line of the Swiss Light Source, Switzerland. Figure 2 shows an image of clearly resolved $10 \mathrm{~nm}$ wide test structures. 

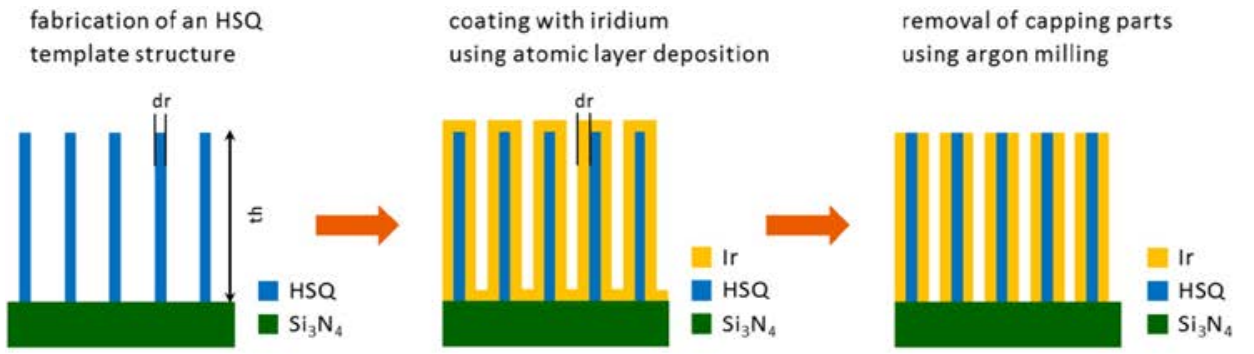

removal of HSQ template using hydrofluoric acid
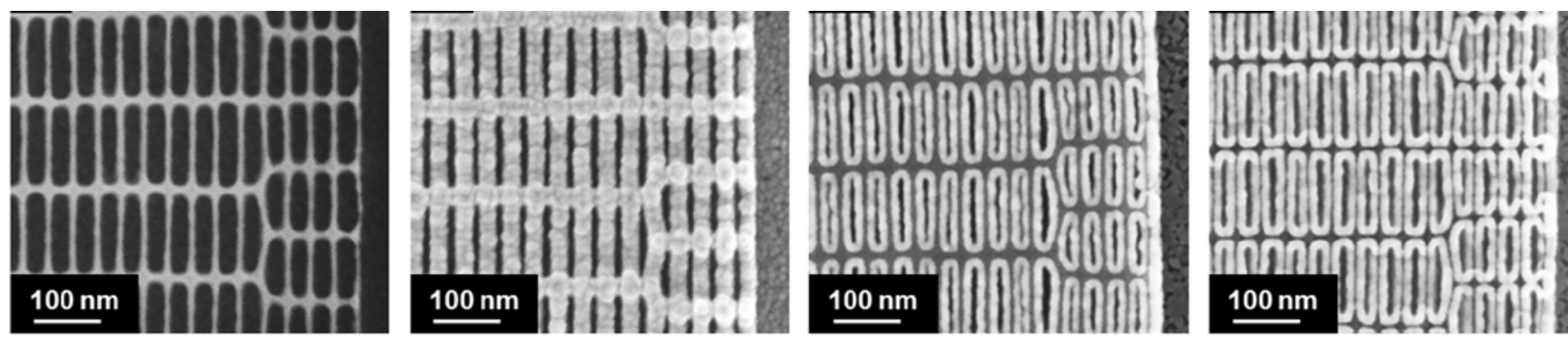

Figure. 1. Top: Fabrication flow of a line-doubled iridium zone plate. Below: corresponding SEM top view images of a zone plate with $8.8 \mathrm{~nm}$ zone width. The structures are $70-80 \mathrm{~nm}$ high.

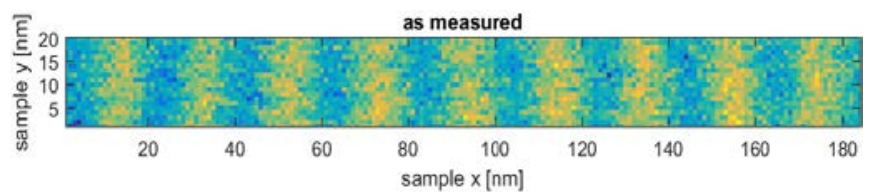

Figure. 2. Soft $x$-ray scanning microscope image of a test object with $10 \mathrm{~nm}$ lines and spaces taken at $850 \mathrm{eV}$ photon energy using a FZP with $8.8 \mathrm{~nm}$ zone width.
Many applications of X-ray optics such as scanning microprobe techniques do not require high resolution in the sub-100 nm range, but rather need the highest possible photon flux in the focal spot. Here, conventional FZPs have the disadvantage that only a small fraction of the incoming photons is diffracted into the used diffraction order. To achieve high diffraction efficiencies, the diffracting zone structures must have a triangular, blazed profile providing a continuous gradient in phase shift from zero to $2 \pi$. In view of the high aspect ratios that are needed to obtain such phase shifts, the fabrication of FZPs with blazed zone profiles is extremely challenging.

As an alternative, we have developed a kinoform diffractive X-ray lens that is based on binary structures [7]. A schematic view is shown in Figure 3a-c. The lens is used in a tilted geometry such that each ray passing through the structures undergoes the correct phase shift for high efficiency focusing. The lens can be used over a wide range of photon energies by adjusting the tilt angle. It is essential to note that the acceptance of the lens is not limited by the height of the diffracting structures. It can therefore be used to collect large X-ray beams. A single lens only focuses in one direction, therefore two devices mounted in orthogonal orientation are used to produce a focal spot.

The lens structures can be fabricated using the so called metal-assisted chemical etching (MACE) [810]. The process is based on producing a gold mask on a silicon membrane substrate by electron-beam lithography and lift-off (Figure 3d). The sample is immersed in a solution of hydrofluoric acid and hydrogen peroxide. The gold structures act as a catalyst enabling the etching of the underlying material, and continuously sinks into the silicon producing structures with very high aspect ratios, see Figure 3e-f. 
a)

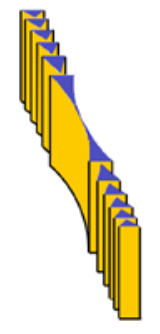

d)

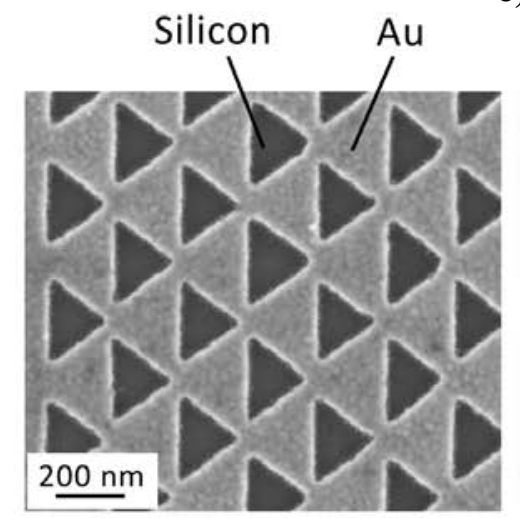

b)

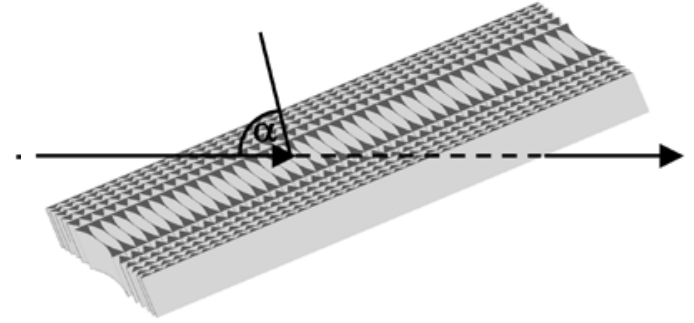

e) c)

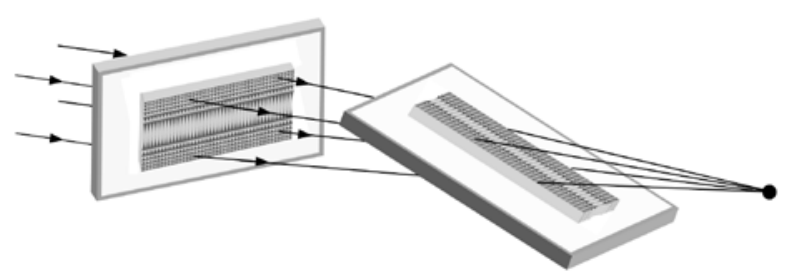

f)
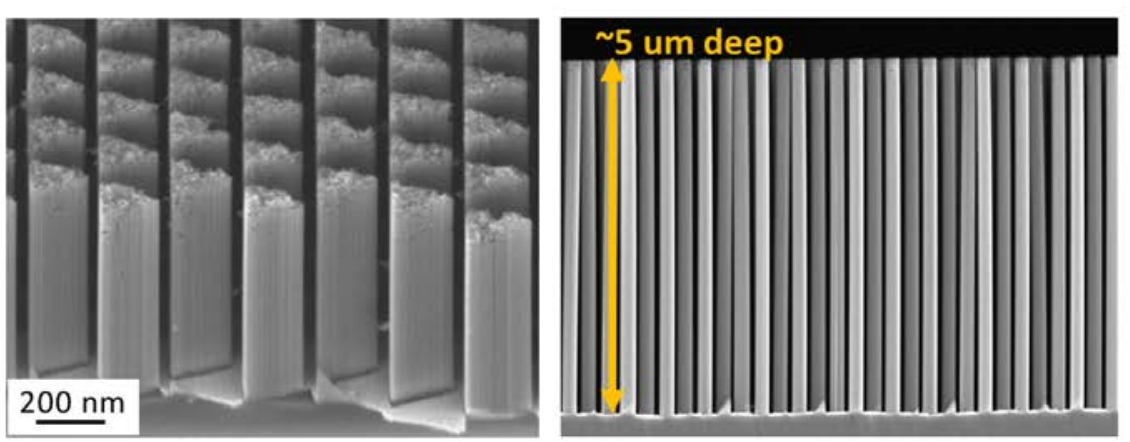

Figure. 3. Top: Schematic view of a kinoform X-ray lens. It consists of individual lenslets that have triangular structures (a). The X-rays are sent through a line of such lenslets under a tilt angle so that every ray passes through a large number of lenslets to undergo the optimum phase shift for high efficiency focusing (b). Two lenses used in orthogonal orientation to produce a focal spot (c). The lens structures can be made from silicon membranes using metal assisted chemical etching (MACE). SEM images of the gold etch mask with $200 \mathrm{~nm}$ pitch (d), tilted view of the triangular lens structures after etching $\sim 1 \mu \mathrm{m}$ deep (e), side view of the final structures (f).

The lenses were tested at the microXAS beam line at the Swiss Light Source and beamline P06 of PETRA III at DESY. We have obtained diffraction efficiencies at $8 \mathrm{keV}$ photon energy of up to $70 \%$ for an individual lens and up to $50 \%$ for focusing into a spot using two lenses, and spot sizes down to 100 $\mathrm{nm}$ have been achieved. As the diffraction efficiency can be controlled via the tilting angle, the technique can also be used to produce hard X-ray beam splitters with tunable splitting ratio [10]. Such devices can be applied for the multiplexing of endstations at XFELs.

Another example of the versatility of diffractive $\mathrm{X}$-ray optics is demonstrated by using an off-axis zone plate as analyzer for resonant inelastic X-ray scattering (RIXS) with good resolving power, as demonstrated at the ADRESS beam line of the Swiss Light Source [11]. Whereas the analyzers based on reflection gratings used in conventional RIXS setups do not convey any information about the nondispersive direction on the detector, a zone plate analyzer provides additional imaging capability as illustrated in Figure 4a. This can be applied to image a line focus on the sample along the nondispersive direction of the detector. We have demonstrated that this can be used to generate a spectrally resolved 2D image by scanning the sample through the line focus [12]. Moreover, by varying the incident photon energy along the line focus, this detection scheme can also be applied to simultaneously collect RIXS spectra for a range of incident energies. An example of this so-called RIXS mapping was demonstrated at beamline P04 of PETRA III at DESY, Hamburg, on a liquid jet of acetonitrile (see Figure 4b). 
a)

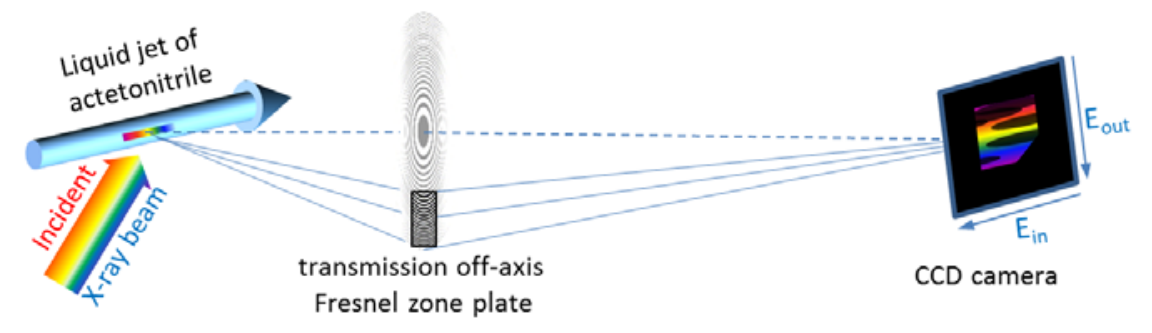

b)

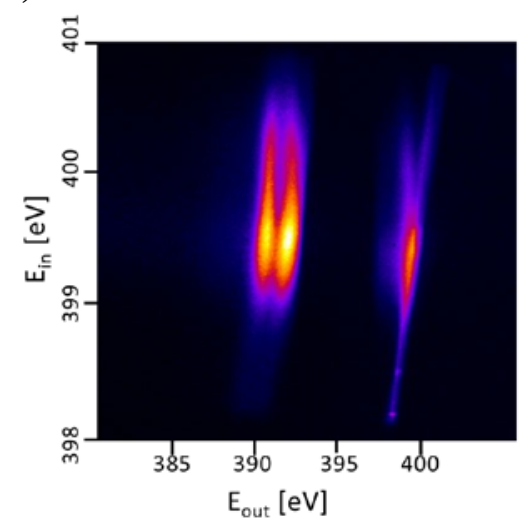

Figure. 4. (a) Schematic view of an off-axis transmission zone plate used as analyzer for resonant inelastic X-ray scattering (RIXS). The imaging properties of a zone plate allow for RIXS mapping, where the incoming photon energy is varied along a line focus on the sample, so that emission spectra can be recorded simultaneously over a range of incident photon energies. (b) RIXS map of acetonitrile.

It should be noted that this scheme of combining the imaging and the dispersive properties of off-axis transmission zone plates is very general, as a multitude of properties can be varied along the line focus on the sample. This may include variations in film thickness, chemical composition, magnetic field, or temperature. In particular in context with XFELs, one can also envision ultrafast time resolved RIXS experiments by varying the arrival time of a pump laser along the non-dispersive direction.

References:

[1] I Vartiainen, et al., Optics Express 23 (2015) p. 13278

[2] B Rösner, et al., Optics Express 25 (2017) p. 30686

[3] K Jefimovs, et al., Physical Review Letters 99 (2007) p. 264801

[4] J Vila-Comamala, et al., Ultramicroscopy 109 (2009) p. 1360

[5] J Vila-Comamala, et al., Optics Express 19 (2011) p. 175

[6] B Rösner, et al., Microelectronic Engineering 191 (2018) p. 91

[7] P Karvinen, et al., Optics Express 22 (2014) p. 16676

[8] X Li and PW Bohn, Applied Physics Letters 77 (2000) p. 2572

[9] C Chang and A Sakdinawat, Nature Communications 5 (2014) p. 4243

[10] M Lebugle, et al., Optics Letters 42 (2017) p. 4327

[11] F Marschall, et al., Optics Express 25 (2017) p. 15624

[12] F Marschall, et al., Scientific Reports 7 (2017) p. 8849 